\section{Vol. 38, Issue 5, October 2011}

\section{Editorial}

285 Immunogenetics: Novel Developments

Garritsen, H.S.P. (Braunschweig); Gassner, C. (Zürich)

Original Articles

287 FOXP3 Expression in GARP-Transduced Helper T Cells Is Not Associated with FOXP3 TSDR Demethylation

Kehrmann, J.; Zeschnigk, M.; Buer, J. (Essen); Probst-Kepper, M. (Braunschweig)

292 The Austrian Bone Marrow Donor Registry: Providing Patients in Austria with Unrelated Donors for Transplant - a Worldwide Cooperation

Rosenmayr, A.; Pointner-Prager, M.; Winkler, M.; Mitterschiffthaler, A.; Pelzmann, B.; Bozic, L.; Pichler-Kurzweil, S.; Tüchler, H.; Fae, I.; Fischer, G.F. (Wien)

\section{Review Articles}

300 Swiss Blood Stem Cells: More than Just a Registry Nicoloso de Faveri, G.; Schwabe, R.; Bart, T. (Bern)

308 What Next? The Next Transit from Biology to Diagnostics: Next Generation Sequencing for Immunogenetics

Gabriel, C.; Stabentheiner, S.; Danzer, M.; Pröll, J. (Linz)

\section{Clinical Information}

\section{Dengue Fever Virus (DENV)}

Arbeitskreis Blut, Untergruppe «Bewertung Butassoziierter Krankheitserreger»

\section{Commentary}

331 To the Understanding of the Statutory Order Empowerment Pursuant to Article 12 German Transfusion Law (Transfusionsgesetz; TFG) and to Guideline Competence Pursuant to Article 12a TFG

von Auer, F (Königswinter)

\section{Band 38, Heft 5, Oktober 2011}

Editorial

285 Immungenetik: Neue Entwicklungen

Garritsen, H.S.P. (Braunschweig); Gassner, C. (Zürich)

Originalarbeiten

287 Die FOXP3-Expression in GARP-transduzierten T-Helfer-Zellen ist nicht mit einer Demethylierung der FOXP3 TSDR assoziiert

Kehrmann, J.; Zeschnigk, M.; Buer, J. (Essen); Probst-Kepper, M. (Braunschweig)

292 Das österreichische Knochenmarkspenderregister: Versorgung von Patienten mit Österreich mit nichtverwandten Spendern für eine Transplantation - eine weltweite Kooperation Rosenmayr, A.; Pointner-Prager, M.; Winkler, M.; Mitterschiffthaler, A.; Pelzmann, B.; Bozic, L.; Pichler-Kurzweil, S.; Tüchler, H.; Fae, I.; Fischer, G.F. (Wien)

\section{Übersichtsarbeiten}

300 Schweizer Blutstammzellen: Mehr als eine Registratur

Nicoloso de Faveri, G.; Schwabe, R.; Bart, T. (Bern)

308 Was kommt denn noch? Next Generation Sequencing in der Immungenetik

Gabriel, C.; Stabentheiner, S.; Danzer, M.; Pröll, J. (Linz)

Klinische Information

318 Dengue-Fieber-Virus (DENV)

Arbeitskreis Blut, Untergruppe «Bewertung Butassoziierter Krankheitserreger»

Kommentar

331 Zum Verständnis der Rechtsverordnungsermächtigung nach $\$ 12$ Transfusionsgesetz (TFG) und der Richtlinienkompetenz nach $\S$ 12a TFG von Auer, F (Königswinter)

\section{KARGER}

Fax +497614520714

Information@Karger.de

www.karger.com 


\section{Vol. 38, Issue 5, October 2011}

Congress Report

334 'Sterility Testing of Blood Components and Advanced Therapy Medicinal Products' (Munich, April 29, 2010) Organized by the DGTI Section 'Safety in Hemotherapy' Meeting Report

Wagner, B.; Grabein, B. (München)

Abstracts

337 'Sterility Testing of Blood Components and Advanced Therapy Medicinal Products' (Munich, April 29, 2010) Organized by the DGTI Section 'Safety in Hemotherapy' Abstracts

341 News / Ticker

344 Meetings and Conferences

291 Imprint

\section{Band 38, Heft 5, Oktober 2011}

Tagungsbericht

334 «Sterility Testing of Blood Components and Advanced Therapy Medicinal Products» (München, 29. April 2010) organisiert durch die DGTI-Sektion «Safety in Hemotherapy» Tagungsbericht

Wagner, B.; Grabein, B. (München)

Abstracts

337 «terility Testing of Blood Components and Advanced Therapy Medicinal Products» (München, 29. April 2010) organisiert durch die DGTI-Sektion «Safety in Hemotherapy» Abstracts

341 News / Ticker

344 Tagungen und Kongresse

291 Impressum

\section{KARGER}

Fax +497614520714 